\title{
New Programming Approach for Steepest Descent Optimization of Rocket Trajectories
}

\author{
Vishnu G Nair \\ Department of Aeronautical \& \\ Automobile Engineering, \\ Manipal Institute of Technology \\ Manipal, India-576104
}

\author{
Dileep M V \\ Department of Instrumentation \& \\ Control Engineering, \\ Manipal Institute of Technology \\ Manipal, India-576104
}

\author{
Prahalad K R \\ Department of Instrumentation \& \\ Control Engineering, \\ Manipal Institute of Technology \\ Manipal, India-576104
}

\begin{abstract}
In this paper a new programming methodology for optimizing rocket trajectories using steepest descent technique is presented. The programming is done in Matlab platform. At first the dynamical equations of rocket is derived and for the proper derivation and analysis of the equations ,Eulers integration method is used. A new programming approach is designed and the equations are optimized using steepest descent technique, assuming certain boundary conditions. The results obtained are verified and it is seen that the optimal trajectory is attained with all objectives satisfied. This project is done at Vikram Sarabhai Space Centre(VSSC), a constituent of Indian Space Research Organization(ISRO).
\end{abstract}

\section{General Terms}

Rocket dynamics,Euler'smethod,Simulator program.

\section{Keywords}

Rocket trajectory,Matlab,Optimization,Steepest descent

\section{INTRODUCTION}

The optimality of rocket trajectories may be defined according to several objectivesliketransfer time is minimal for a given propellant and payload mass (i.e. for a given launch mass), the required propellant mass is minimal for a given transfer time and launch mass, the required propellant mass is minimal for a given transfer time and payload mass.In practice, the methods of solving deterministic optimal control problems are divided into two categories: direct and indirect methods[9]. Indirect methods proceed by formulating the optimality conditions according to the Pontryagin maximum principle and then numerically solving the resulting two point boundary value problem. Direct methods discretize the original problem in time and solve the resulting parameter optimization problem and thus generate an approximate solution of the original problem. Both methods solve the necessary conditions of optimality and both discretize the problem. The most common objective is to minimize the propellant required or equivalently to maximize the fraction of the rocket that is not devoted to propellant. Of course, as is common in the optimization of continuous dynamical systems, it is usually necessary to provide some practical upper bound for the final time or the optimizer will trade time for propellant. There are also rocket trajectory problems where minimizing flight time is an important, or, for example those using continuous thrust, where minimizing flight time and minimizing propellant use are synonymous.

\section{MATHEMATICAL REPRESENTATION OF ROCKETDYNAMICS}

Point-mass equations of motion with non-rotating sphericalearth are considered in this paper, which is given by

$$
\begin{gathered}
\dot{r}=V \sin \gamma \\
\dot{V}=\frac{1}{m V}(T \cos \alpha--m g \sin \gamma) \\
\dot{\gamma}=\frac{1}{m V}(T \sin \alpha)+\left(\frac{V}{r}-\frac{g}{V}\right) \cos \gamma
\end{gathered}
$$

For a problem without path constraints, with terminal equality constraints, and with an initial state vector whose components are either fixed or optimizable, the first order necessary conditions can be formulated as shown in equations from(4)to(11).

Hamiltonian:

$$
H=L+\lambda^{T} f
$$

Dynamics:

$$
\dot{X}=f(x, u, t)=\left(\frac{\partial H}{\partial \lambda}\right)^{T}
$$

Adjoint differential equations:

$$
\dot{\lambda}=\left(\frac{\partial L}{\partial x}\right)^{T}-\left(\frac{\partial f}{\partial x}\right)^{T} \lambda=\left(\frac{\partial H}{\partial x}\right)^{T}
$$

Optimality condition:

$$
\left(\frac{\partial H}{\partial u}\right)^{T}=0=\left(\frac{\partial f}{\partial u}\right)^{T} \lambda+\left(\frac{\partial L}{\partial x}\right)^{T}
$$

Initial conditions:

$$
x_{k}\left(t_{0}\right) \text { given or } \lambda_{k}\left(t_{0}\right)=0
$$

Terminal constraints:

$$
\Psi_{f}\left(x_{f}, t_{f}\right)=0
$$


Transversality conditions:

$$
\lambda_{f}=\left(\frac{\partial \emptyset}{\partial x}+v^{T} \frac{\partial \Psi_{f}}{\partial x}\right)_{t=t_{f}}
$$

Transversality condition for optimizablet :

$$
\Omega=\left[\left(\frac{\partial \emptyset}{\partial t}+v^{T} \frac{\partial \Psi_{f}}{\partial t}+H\right)\right]_{t=t_{f}}
$$

Where $\mathrm{L}=$ Lagrangefunction

$$
\begin{aligned}
& H=\text { Hamilton function } \\
& \lambda=\text { costate variable }
\end{aligned}
$$

Euler-Lagrange [22] equations were extended by the introduction of further necessary conditions, such as the Legendre- Clebsch condition, The Jacobi condition[4] and the Weierstrass condition[4].

$$
\frac{\partial^{2} H}{\partial u^{2}} \geq 0
$$

A detailed discussion on these conditions can be found in Ref. [22].In this part optimization of the three variables take place, they are radial distance from the center of earth $r$, rocket velocity $v$ and the flight path angle $\gamma$. The equations (1) - (3) can be described in generic form as,

$$
\begin{gathered}
\dot{X}=f(X, U) \\
{\left[\begin{array}{c}
\dot{r} \\
\dot{v} \\
\dot{\gamma}
\end{array}\right]=\left[\begin{array}{c}
V \sin \gamma \\
\frac{1}{m V}(T \cos \alpha-m g \sin \gamma) \\
\frac{1}{m V}(T \sin \alpha)+\left(\frac{V}{r}-\frac{g}{V}\right) \cos \gamma
\end{array}\right]}
\end{gathered}
$$

Where the state and control vectors are defined as

$$
\dot{X}=\left[x_{1} x_{2} x_{3}\right]^{T}
$$

That is

$$
\begin{gathered}
\dot{X}=[r v \gamma]^{T} \\
U=\alpha
\end{gathered}
$$

In this a trajectory optimization problem of a single stage launch vehicle is considered. The objective here is to generate the guidance command history $\alpha(t), t \in\left(t_{0}, t_{f}\right)$ such that the following concerns are taken care of,

(a) At the final time $t_{f}$, the specified terminal constraints must meet as accurately. The terminal constraints include constraints on altitude, velocity and flight path angle (which is the angle made by the velocity vector with respect to the local horizontal). (b) The system should demand minimum guidance command, which can be ensured by formulating a 'minimum time' problem.

To achieve the above objectives, the following cost function is selected, which consists of terminal penalty terms and a dynamic control minimization term.

$$
\begin{aligned}
J=\left(r\left(t_{f}\right)-r_{f}\right)^{2} s_{r} & +\left(v\left(t_{f}\right)-v_{f}\right)^{2} s_{v} \\
& +\left(\operatorname{row}\left(t_{f}\right)-\operatorname{row}_{f}\right)^{2} s_{\gamma}
\end{aligned}
$$

Where $s_{r}, s_{v}$, and $s_{\gamma}$ weighing factors. The Hamiltonian (Ref equation (4)) is defined as ,

$$
H=J+\lambda^{T}
$$

Where $\lambda^{T}=\left[\lambda_{1} \lambda_{2} \lambda_{3}\right]$, costate variables.

$$
\begin{aligned}
H=J-\lambda_{1}(V \sin \gamma) & -\lambda_{2}\left(\frac{1}{m V}(T \cos \alpha-m g \sin \gamma)\right) \\
& -\lambda_{3}\left(\frac { 1 } { m V } ( T \operatorname { s i n } \alpha ) \left(\frac{V}{r}\right.\right. \\
& \left.\left.-\frac{g}{V}\right) \cos \gamma\right)
\end{aligned}
$$

From equation (18) we get

$$
\begin{aligned}
H=\left(r\left(t_{f}\right)-r_{f}\right)^{2} s_{r} & +\left(v\left(t_{f}\right)-v_{f}\right)^{2} s_{v} \\
+ & \left(\operatorname{row}\left(t_{f}\right)-\operatorname{row}_{f}\right)^{2} s_{\gamma}-\lambda_{1}(V \sin \gamma) \\
& -\lambda_{2}\left(\frac{1}{m V}(T \cos \alpha-m g \sin \gamma)\right) \\
& -\lambda_{3}\left(\frac{1}{m V}(T \sin \alpha)\right. \\
+ & \left.\left(\frac{V}{r}-\frac{r}{V}\right) \cos \gamma\right)
\end{aligned}
$$

Equation (21) gives the final Hamiltonian function. This function has solve to get the optimum solution .Therefore

$$
\frac{\partial H}{\partial r}=\lambda_{3} \frac{v}{r^{2}} \cos \gamma=-\dot{\lambda_{1}}
$$

$$
\begin{gathered}
\frac{\partial H}{\partial v}=-\lambda_{1} \sin \gamma+\lambda_{3}-\left(\frac{1}{m v^{2}}(T \sin \alpha)-\left(\frac{1}{r}-\frac{g}{v^{2}}\right) \cos \gamma\right) \\
=-\dot{\lambda}_{2}
\end{gathered}
$$

$\frac{\partial H}{\partial \gamma}=-\lambda_{1} \cos \gamma-\lambda_{2} g \cos \gamma+\lambda_{3}\left(\frac{v}{r}-\frac{g}{v}\right) \sin \gamma$

$$
=-\dot{\lambda_{3}}
$$

$\frac{\partial H}{\partial \alpha}=\lambda_{2} \frac{T}{m} \sin \alpha-\lambda_{3} \frac{T}{m v} \cos \alpha$

The above equations $(22)-(25)$ to be solved to get the solutions of costate variables, that is $\lambda_{1}, \lambda_{2}$ and $\lambda_{3}$. In an optimal control formulation, the necessary conditions of optimality include the state equation, costate equation, optimal control equation and appropriate boundary conditions.An steepest descent method needs accurate initial 
conditions. The equations (1)(2)\&(3) in the simulator program is developed initial steering profile for steepest descent optimization is obtained.For finding the accurate steering profile taking an assumption that is acceleration is a linearly increasing quantity. So rate of change of velocity is approximating as linear function. This linear function is selected by polynomial approximation[17] that is by changing the functions randomly to fit the curve properly.

$$
\alpha=\cos ^{-1}\left(\frac{c_{1} t+c_{2}+g \sin \gamma}{T / m}\right)
$$

Now knowing the values of $\dot{\lambda}_{1}, \dot{\lambda}_{2}$ and $\dot{\lambda}_{3}$, so to get $\lambda_{1}, \lambda_{2}$ and $\lambda_{3}$ have to integrate. Therefore the final values of $\lambda^{T}$ is $\lambda_{1 f}, \lambda_{2 f}$ and $\lambda_{3 f}$. To find the final values of $\lambda$.

$$
\begin{aligned}
& \lambda_{1 f}=\frac{\partial J}{\partial r}=2 \times s_{r} \times\left(r\left(t_{f}\right)-r_{f}\right) \\
& \lambda_{2 f}=\frac{\partial J}{\partial v}=2 \times s_{v} \times\left(v\left(t_{f}\right)-v_{f}\right) \\
& \lambda_{3 f}=\frac{\partial J}{\partial \gamma}=2 \times s_{\gamma} \times\left(\gamma\left(t_{f}\right)-\gamma_{f}\right)
\end{aligned}
$$

The integration method used in the optimal control problem is Eulers method. It is the usual basic method.

\subsection{Implementation of Euler's method}

Euler's method can be implemented both in simulator program and the optimal control problem. In simulator program the integration of $r, v$ and $\gamma$ happens. That is given as follows.

$$
\begin{gathered}
r=r_{0}+\dot{r} d t \\
v=v_{0}+\dot{v} d t \\
\gamma=\gamma_{0}+\dot{\gamma} d t
\end{gathered}
$$

In optimal control the $\lambda^{T}$ is integrating to get final $\lambda$ values.

$$
\begin{aligned}
& \lambda_{1}=\lambda_{10}+\dot{\lambda_{1}} d t \\
& \lambda_{2}=\lambda_{20}+\dot{\lambda_{2}} d t \\
& \lambda_{3}=\lambda_{30}+\dot{\lambda_{3}} d t
\end{aligned}
$$

Where $d t$ is the integration step size. $r_{0}, v_{0} \& \gamma_{0}$ are the initial values of $r, v$ and $\gamma$. Similarly $\lambda_{10}, \lambda_{20} \& \lambda_{30}$ are the initial values of $\lambda_{1}, \lambda_{2} \& \lambda_{3}$. Thus gets the solution of equation (21) For optimal solutions, The optimality condition (Ref equation (26)) is given by

$$
\frac{\partial H}{\partial \alpha}=0
$$

The weighting factor tow $(\tau)$ should select according to the steepest descent method. That is

$$
\begin{aligned}
& \text { toww }=\frac{\partial H}{\partial \alpha} \times\left(\frac{\partial H}{\partial \alpha}\right)^{T} \\
& \operatorname{tow}(\tau)=\frac{1}{\sqrt[2]{\text { tow } w}}
\end{aligned}
$$

Thus weighting factor tow $(\tau)$ is obtained. Then it is applied to the control variable alpha $(\alpha)$,toget the new alphaprofile.

$$
\alpha=\alpha_{0}-\frac{\partial H}{\partial \alpha} \times \operatorname{tow}(\tau)
$$

Where $\alpha_{0}=$ privious computed $\alpha$

Equation (21) is the gradiant function, optimizes the objective function. An optimal control problem needs accurate initial conditions. From the equations (1) - (3) the simulator program is developedand initial steering profile for steepest descent optimization is obtained.For finding the accurate steering profile acceleration is assumed to be a linearly increasing quantity. So rate of change of velocity is approximated as linear function. This linear function is selected by polynomial approximation that is by changing the functions randomly to fit the curve properly. The vehicle acceleration is a linear function of time as given in the equation. The constants are obtained by varying initial and final steering angle value. Thus tuning of constants $c_{1}$ and $c_{2}$ are done. The initial and final $\alpha$ is thus obtained to be 3.5 deg of and $14 \mathrm{deg}$ respectively.Therefore by solving the equation (21)using the initial and final values the appropriates values for $c_{1}$ and $c_{2}$ can be find out;

$$
\begin{gathered}
c_{1}=6.4217 \times 10^{-3} \\
c_{2}=1.918235
\end{gathered}
$$

\section{SIMULATOR PROGRAM STEPS AND RESULTS}

The simulator program is mainly used for generating the initial steering angle. This steering is given as the input thrust angle for trajectory optimization problem. Table (1)shows travelling time and the initial steering angle profile. The main programming steps are given below:-

a)-Develop rocket dynamics equations.

b)-To get initial alpha profile develop a simulator program that has minimum objective function values.

c)-Put this alpha profile in the trajectory optimization problem.

d)By varying costate variables optimization takes place 
e)Alpha profile is updated by using steepest descent technique.

f) Alpha profile update is done by tuning of multiplication factor.

g) The optimized result will be obtained after 82 iterations.

The results obtained are shown below:-

Table 1 Travelling time and the initial steering angle profile

\begin{tabular}{|l|l|}
\hline $\begin{array}{l}\text { Time } \\
\text { seconds }\end{array}$ & $\begin{array}{l}\text { initial thrust angle } \\
\alpha \text { values in radian }\end{array}$ \\
\hline 1 & 0.275101 \\
\hline 50 & 0.279912 \\
\hline 100 & 0.271316 \\
\hline 150 & 0.255160 \\
\hline 200 & 0.235788 \\
\hline 250 & 0.217232 \\
\hline 300 & 0.203625 \\
\hline 350 & 0.198925 \\
\hline 400 & 0.206037 \\
\hline 450 & 0.226118 \\
\hline 500 & 0.258920 \\
\hline 510 & 0.266945 \\
\hline $540 w 5$ & $0659 n e d$ \\
\hline
\end{tabular}

Figure (1) shows the graph obtained for the simulation program.The figure indicates the radial distance from earth centre. The symbol, ${ }^{*}$, indicates the target, $r f=$ $6862.41573 \mathrm{~km}$. By this method, the rocket achieved the desired position, but not the desired velocity. Therefore the above method is not an optimized one. So has to be optimized.

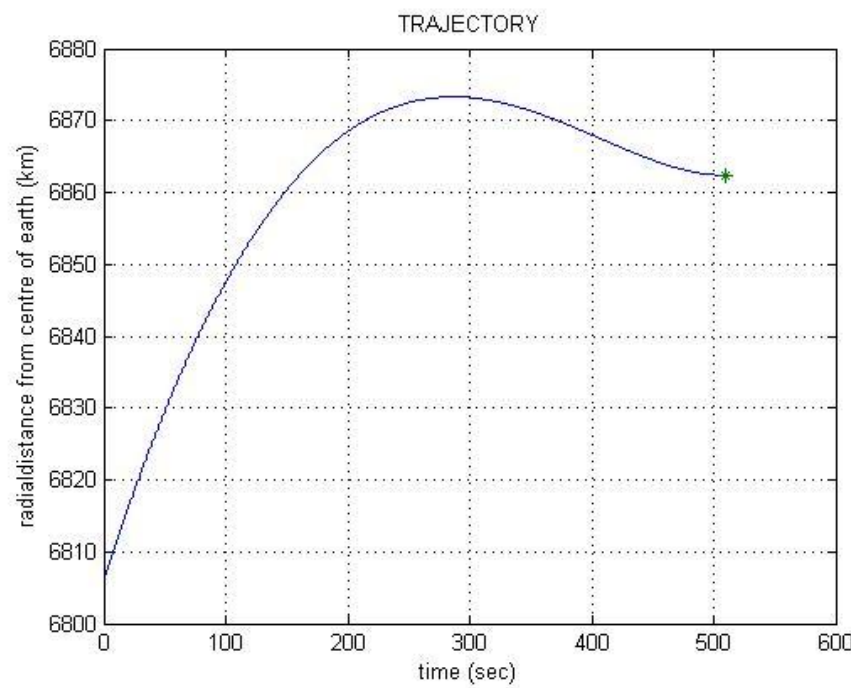

Fig 1 Radial distance from earth centreVs time

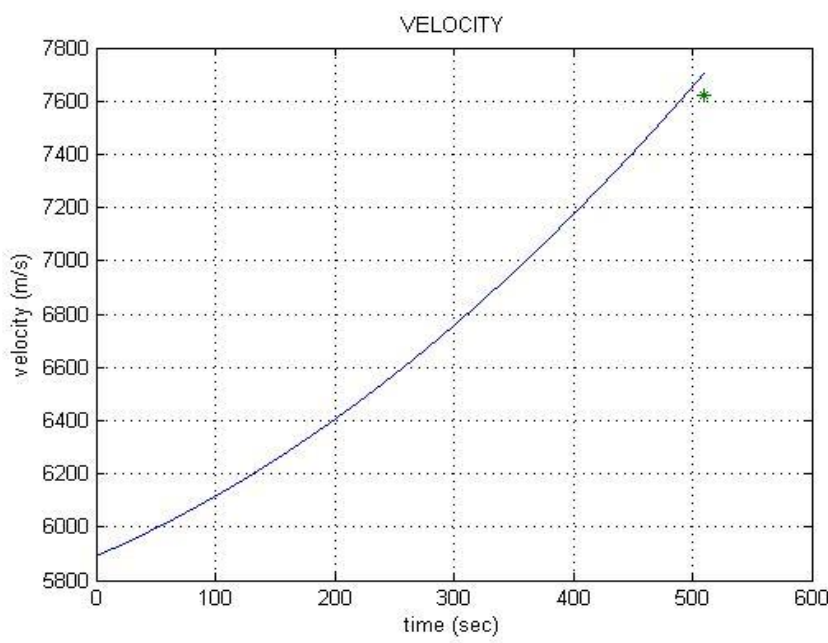

Fig 2 Velocity Vs time

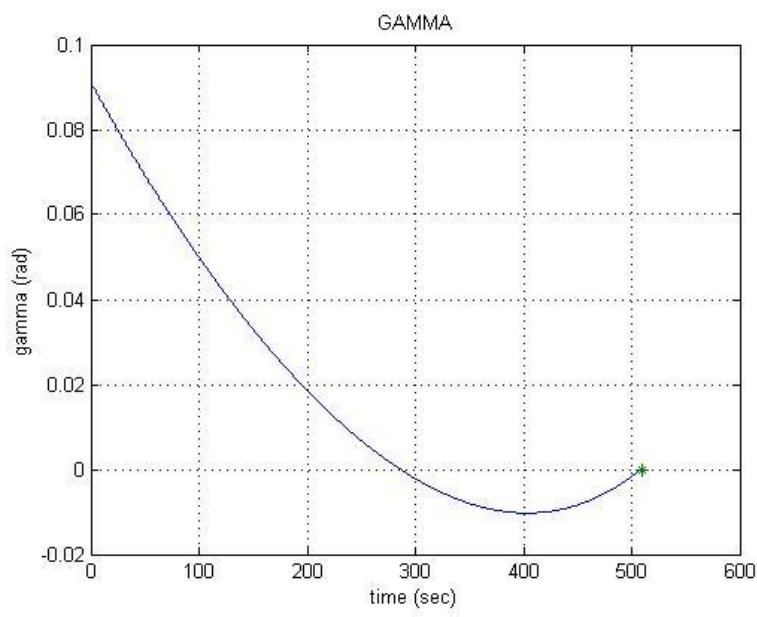

Fig 3: Flight path angle Vs time

Figure (3)\&(2) indicates the flight path angle and velocity variation with respect to time.1n figure (4.8), the symbol' *' indicates the value of final velocity, $V f=7623.5321 \mathrm{~m} / \mathrm{s}$ 
and in figure (3), it indicates the value of final gamma, $\gamma=$ $0 \mathrm{deg}$. In figure (2) the desired target-velocity is not achieved. The simulation program provides the basic model trajectory and the initial steering angle. The trajectory optimization problem is explained in the following sections. Euler's method of integration can be used for optimization. In Euler's method of integration the integration step size is same. Iteration results are shown below. Total iteration number $=$ 82

Table 2 Itration number and the objective function value

\begin{tabular}{|c|c|}
\hline $\begin{array}{l}\text { Iteration } \\
\text { number }\end{array}$ & $\begin{array}{l}\text { Objective } \\
\text { function values }\end{array}$ \\
\hline 1 & 64.0930 \\
\hline 5 & 93581 \\
\hline 10 & 22177 \\
\hline 15 & 76128 \\
\hline 20 & 19333 \\
\hline 25 & 53712 \\
\hline 30 & 20685 \\
\hline 35 & 28653 \\
\hline 40 & 20762 \\
\hline 45 & 16666 \\
\hline 50 & 12255 \\
\hline 55 & 81863 \\
\hline 60 & 4699.7 \\
\hline 65 & 2037.8 \\
\hline 70 & 418.6931 \\
\hline 71 & 237.3277 \\
\hline 73 & 30.6289 \\
\hline 75 & 26.113 \\
\hline 77 & 2.289 \\
\hline 79 & 0.0002527 \\
\hline
\end{tabular}

\begin{tabular}{|l|l|}
\hline 80 & $8.3802 \times 10^{-6}$ \\
\hline 81 & 0.0002527 \\
\hline 82 & $8.3802 \times 10^{-6}$ \\
\hline
\end{tabular}

Table (2) shows the objective function values for each iteration. By analyzing the objective function values, it seems to be decreasing and tends to a minimum value. Table (3) shows final radial distance from center of earth, final velocity of rocket and final flight path angle in each iteration.

Table 3 Iterationnumber, final radial distance, final velocity and final flight path angle

\begin{tabular}{|c|c|c|c|}
\hline $\begin{array}{l}\text { Iteration } \\
\text { number }\end{array}$ & $\begin{array}{l}\text { final radial } \\
\text { distance from } \\
\text { centre of earth } \\
(\mathrm{km})\end{array}$ & $\begin{array}{l}\text { final velocity } \\
\text { of rocket } \\
(\mathrm{m} / \mathrm{s})\end{array}$ & $\begin{array}{l}\text { Final flight } \\
\text { path } \\
\text { angle }(\mathrm{rad})\end{array}$ \\
\hline 1 & 6862.4 & 7703.6 & 0.0002116 \\
\hline 5 & 68655 & 7696.6 & 0.0015444 \\
\hline 10 & 6863.9 & 7700.7 & -0.0002827 \\
\hline 15 & 6865.2 & 7697.5 & -0.0004971 \\
\hline 20 & 6863.8 & 7700.2 & -0.0024106 \\
\hline 25 & 6864.7 & 7696.5 & -0.0030378 \\
\hline 30 & 6863.9 & 7696.0 & -0.0049032 \\
\hline 35 & 6864.1 & 7691.4 & -0.0061224 \\
\hline 40 & 6863.9 & 7686.7 & -0.0076483 \\
\hline 45 & 6863.7 & 7680.5 & -0.0090698 \\
\hline 50 & 6863.5 & 7673.3 & -0.010482 \\
\hline 55 & 6863.3 & 7664.9 & -0.011885 \\
\hline 60 & 6863.1 & 7655.5 & -0.013288 \\
\hline 65 & 6862.9 & 7645.0 & -0.014698 \\
\hline 70 & 6862.6 & 7633.5 & -0.016121 \\
\hline 71 & 6862.6 & 7631.0 & -0.016407 \\
\hline 73 & 6862.5 & 7626.1 & -0.016981 \\
\hline 75 & 6862.4 & 7623.6 & -0.01713 \\
\hline
\end{tabular}




\begin{tabular}{|l|l|l|l|}
\hline 77 & 6862.4 & 7623.6 & -0.017293 \\
\hline 79 & 6862.4 & 7623.5 & -0.017286 \\
\hline 80 & 6862.4 & 7623.5 & -0.017286 \\
\hline 81 & 6862.4 & 7623.5 & -0.017286 \\
\hline 82 & 6862.4 & 7623.5 & -0.017286 \\
\hline
\end{tabular}

\begin{tabular}{|l|l|}
\hline 80 & 0.011844 \\
\hline 81 & 0.001649 \\
\hline 82 & 0.011843 \\
\hline
\end{tabular}

Table (4) shows weighting factor $(\tau)$ variation in each iteration. Weighting factor has a significant major role in optimizing the trajectory. Figure(4), (5) \& (6) shows the graphs obtained for Steepest Descent method with Euler's integration. The figure (4)indicates the radial distance from earth center. The symbol, ' $*$ ' indicates the target, $r f=$

$6862415.73 \mathrm{~m}$. The above method is optimized with minimum objective function value. From the graph it can be seen that the rocket achieved the target-position.A higher altitude is considered in this method, sothat it can achieve the

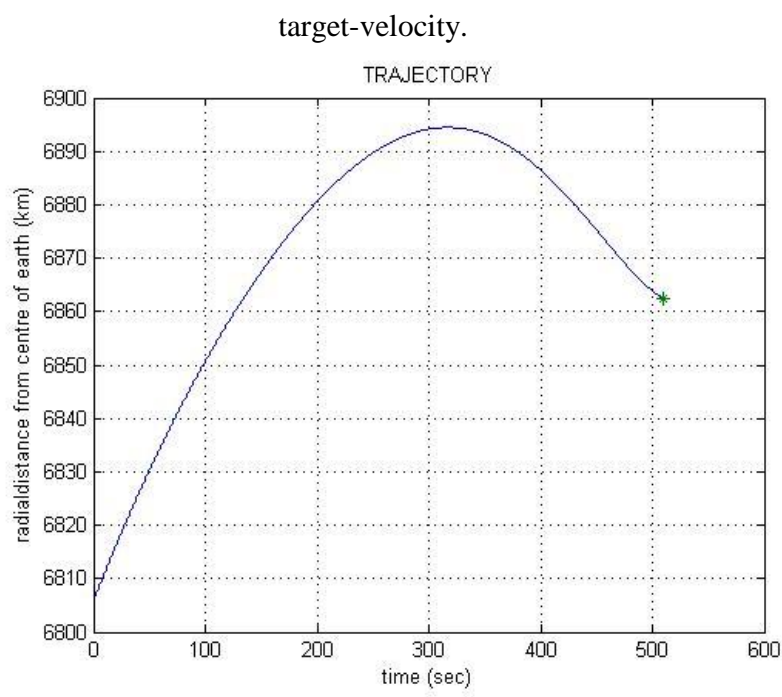

Fig.4 Radial distance from the centre of earth Vs time

Figure (5)\&(6)indicates the flight path angle and velocity variation with respect to time.1n figure (5)the symbal * ' indicate the final velocity, $V f=7623.5321 \mathrm{~m} / \mathrm{s}$ and in figure (6)the symbal *' indicates final gamma , $\gamma=$ $0.0002 \mathrm{deg}$. In figure (4), graph achieving the target-velocity while comparing with the simulator program results (refer figure (2)).The graph shows that the desired gamma value is not achieved. But it is the optimal solution since the objective function has the minimum value. 


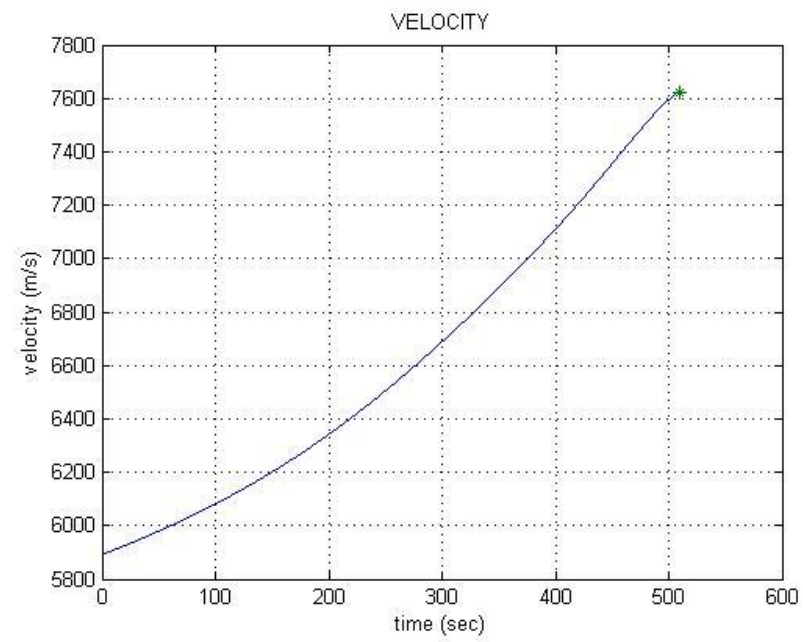

Fig 5 VelocityVstime

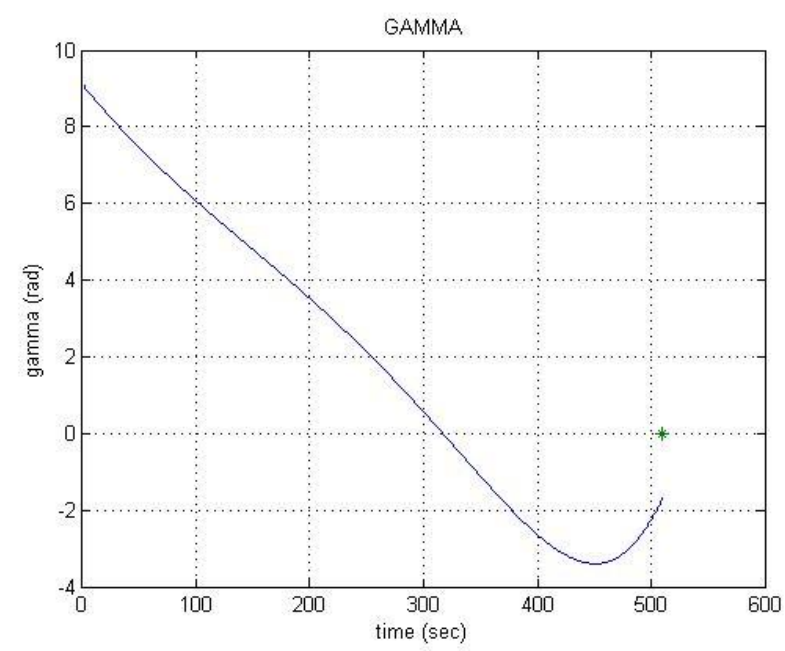

Fig .6 Flight path angle Vs time

\section{5 .CONCLUSIONS}

A new programming approach for steepest descent optimization of rocket trajectories is presented in this paper. From the results it is clear that the final objectives are achieved with optimum resources and time. All the constraints are satisfied and the trajectory is a optimal one. This methodology can be implemented in future missions and the results ]can be verified and compared with the real time outputs. Comparison of the results with real time results and formulation of more efficient algorithms will be the future work.

\section{6 .ACKNOWLEDGMENTS}

The authors would like to express their appreciation to Dinesh Kumar, Scientist grade D , of Indian Space Research Organization (ISRO) for his sensible help and honourable support.

\section{REFERENCE}

[1]John T. Betts "Survey of Numerical Methods for Trajectory Optimization", AIAA Journal of Guidance, Control and Dynamics, Vol. 21, No. 2, March-April 1998, pp. 193-207.
[2] Betts, J. T., and Frank, P. D., "A Sparse Nonlinear Optimization Algorithm," Journal of Optimization Theory and Applications, Vol. 82, 1994, pp. 519-541.

[3]Peter F. Gath, Klaus H. Well "Trajectory Optimization Using A Combination of Direct Multiple Shooting"AIAA Guidance, Navigation, and Control Conference and Exhibit 6-9 August 2001 Montreal, Canada.

[4]Peter Friedrich Gath "CAMTOS - A Software Suite Combining Direct and Indirect Trajectory Optimization Methods" Institute of Flugmechanik und Flugregelung University Stuttgart 2002.

[5]LukkanaVaraprasad and RadhakantPadhi "Ascent Phase Trajectory Optimization Of a Genetic Launch Vehicle " XXXII NATIONAL SYSTEMS CONFERENCE, NSC 2008, December 17-19, 2008

[6]JukkaRanta“optimal control and flight trajectory optimization applied to evasion analysis"Espoo, March 2004

[7]Siddal, J.N.,“ Optimal Engineering Design: Principles and Applications”, Mercell Dekker Inc., New York, NY, 1982.

[8]Bryson, A. E. and Denham, W. F., "A Steepest-Ascent Method for Solving Optimum Programming Programs," Journal of Applied Mechanics, Vol. 29, pp. 247-257, June 1962.

[9]Fletcher, R., "Practical Methods of Optimization," Vol. 2, ConstrainedOptimization, Wiley, New York, 1985.

[10]Gilberto E. Urroz, "Solution of non-linear equations" September 2004

[11]Bruce A. Conway "Spacecraft Trajectory Optimization"Cambridge University Press, (C) Cambridge University Press 2010ISBN-13 978-0-52151850-5

[12]Donald Greenspan “ Numerical Solution of Ordinary Differential Equations for Classical, Relativistic and Nano Systems. " Copyright (C) 2006 WILEY-VCH Verlag GmbH \& Co. KGaA, Weinheim, ISBN: 3-527-40610-7

[13]Ulrich Walter"Astronautics" ( $) 2008$ WILEY-VCH Verlag GmbH \& Co. KgaA, Weinheim ISBN:978-3-527-40685-2

[14]Mischa Kim"Continuous Low-Thrust Trajectory Optimization: Techniques And Application” (C) 2005 by Mischa Kim

[15]Desineni Rama Naidu "Optimal control Systems"Boca Raton London New York Washington, D.C. (C) 2003 by CRC Press LLC ISBN 0-8493-0892-5

[16]Bryson, A.E., Jr., Ho, Y.-C., "Applied Optimal Control”, Revised Printing, Hemisphere Publishing Corporation, 1975

[17]Gordon K. Smyth "Polynomial Approximation"John Wiley \& Sons, Ltd, Chichester, 1998 ,ISBN 0471975761

[18]John H. Mathews and Kurtis K. Fink "Numerical Methods Using Matlab", 4th Edition, 2004ISBN: 0-13-065248-2

[19]“THE ART OF SCIENTIFIC COMPUTING "Copyright (C) 1986-1992 by Cambridge University Press. Programs Copyright (C) 1986-1992 by Numerical Recipes Software. ISBN 0-521-43064-X 